\title{
Graphene synthesis: a Review
}

\author{
S. Saqib Shams*, Ruoyu Zhang, Jin Zhu \\ Ningbo Key Laboratory of Polymer Materials, Ningbo Institute of Industrial Technology, Chinese Academy of Sciences, \\ Ningbo, Zhejiang 315201, P. R. China
}

\begin{abstract}
Graphene has achieved a great amount of popularity and interest from the science world because of its extraordinary physical, mechanical and thermal properties. Graphene is an allotrope of carbon, having one-atom-thick planar sheets of $\mathrm{sp}^{2}$ bonded carbon atoms densely packed in a honeycomb crystal lattice. Many methods to synthesize graphene have been developed over a short period and we believe it is necessary to create a list of the most notable approaches. This article focuses on the methods to synthesize graphene in an attempt to summarize and document advancements in the synthesis of graphene research and future prospects.
\end{abstract}

Keywords: graphene; synthesis; nanomaterials; graphite; 2D; biomass

(C) Wroclaw University of Technology.

\section{Introduction}

Graphene is perhaps the simplest form of carbon and definitely the thinnest material ever produced. It has one-atom-thick planar sheets of $\mathrm{sp}^{2}$ bonded carbon atoms densely packed in a honeycomb crystal lattice [1]. It is useful to understand graphene as a few layers of graphite. In this context, the extraordinary properties of honeycomb carbon are not really surprising. In middle ages, the layered morphology and weak dispersion forces between adjacent sheets were utilized to make marking instruments, similar to our use of pencils today. Recently, graphite is being used as dry lubricant, instead of lavishly priced hexagonal boron nitride and molybdenum disulphide [2]. Graphite is also used in electrodes because of high in-plane electrical conductivity $\left(\sim 10^{4} / \Omega \cdot \mathrm{cm}\right)$ and in industrial blast furnaces because of its excellent thermal conductivity $(\sim 3000 \mathrm{~W} / \mathrm{m} \cdot \mathrm{K})[3,4]$. The term 'graphene' was first used by Boehm et al. [5], while describing single layer carbon foils. It was derived from graphite and the suffix '-ene'. Graphite flakes are basically a stack of graphene layers with an interplanar spacing of $3.5 \AA$ [6] and having $\mathrm{C}-\mathrm{C}$ bond length of about $0.142 \AA$ [7]. Like

\footnotetext{
*E-mail: s.saqib.shams@gmail.com
}

explained by Geim et al. [8], "graphene is a single atomic plane of graphite, which - and this is essential - is sufficiently isolated from its environment to be considered free-standing". Graphene strikes as a remarkable candidate for making functional nanocomposites having excellent properties. The fact that its precursor (graphite) is readily available makes it all the more tempting [9]. Defectfree graphene presents outstanding physical properties, such as high intrinsic mobility and ballistic transport, high thermal conductivity and Young's modulus, an optical transmittance of almost $98 \%$ and a large specific surface area $[1,10,11]$. Much attention has been given to the characterization of graphene and its derivatives so we will not be discussing the properties of graphene. This review article focuses on the brief history and recent advancement in synthesis of graphene.

\section{Synthesis of graphene}

Graphene has been in the limelight for some time now and many researchers have been working on graphene synthesis, thus, several methods have been reported for the exfoliation of graphite into graphene. They can be divided into two main categories: the top-down approach and the bottom-up approach. Graphite is simply a stack 


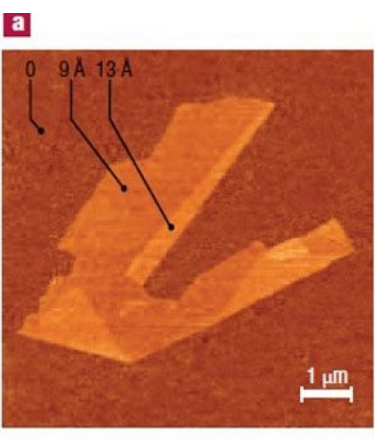

๒

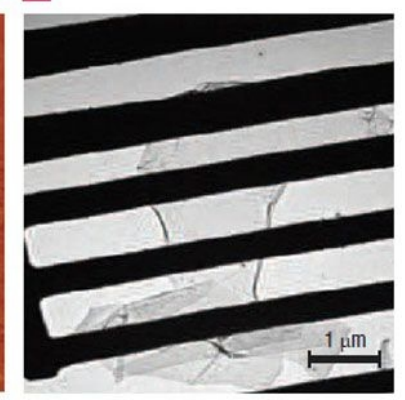

E

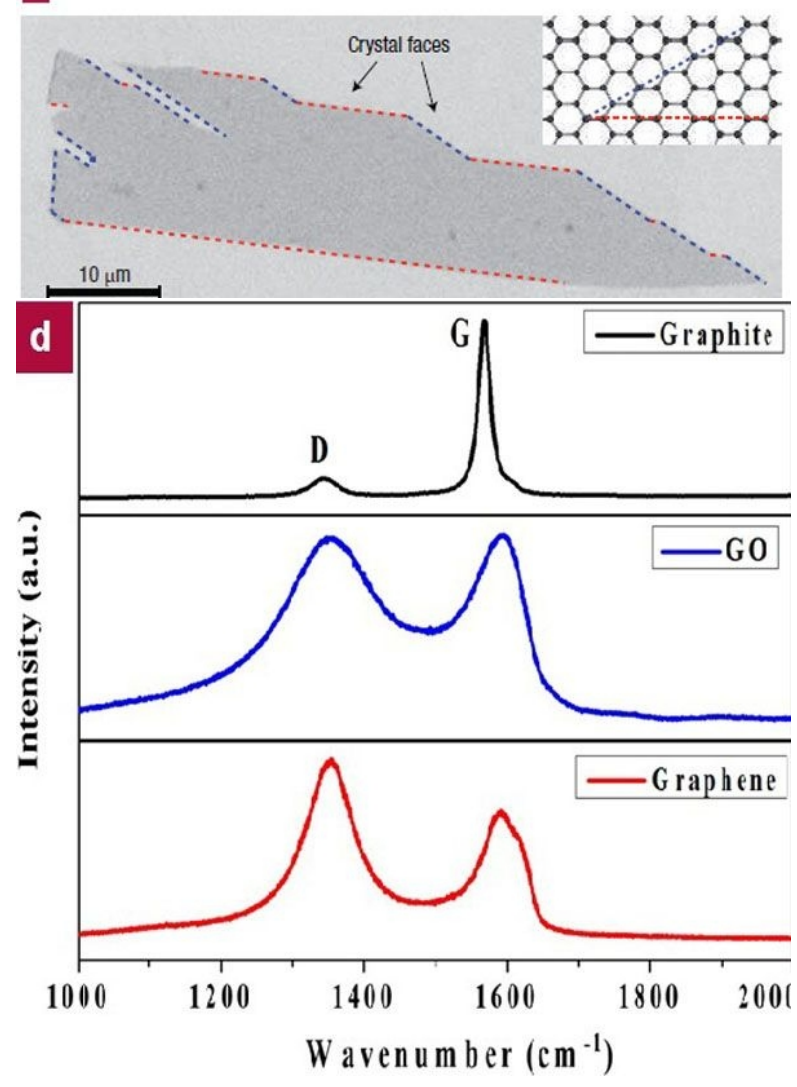

Fig. 1. (a) AFM image of graphene. The folded region exhibiting a relative height of $\sim 4 \AA$ clearly indicates that it is a single layer (Copyright National Academy of Sciences, USA). (b) A graphene sheet freely suspended on a micrometer-size metallic scaffold. (c) Scanning electron micrograph of a relatively large graphene crystal, which shows that most of the crystal's faces are zigzag and armchair edges as indicated by blue and red lines and illustrated in the inset. 1D transport along zigzag edges and edgerelated magnetism are expected to attract significant nano-tubes attention [1]. (d) Raman spectra of graphite, GO and graphene sheets [12]. (reprinted with permission). of graphene layers and to be able to separate these layers into individual sheets of graphene, van der Waals forces need to be overcome [13]. This approach is referred to as the top-down approach. Several challenges are associated with this approach, like surface defects that occur during sheet separation and the separated sheets reagglomerating afterwards. In general, the top-down approaches offer low yields and are tedious procedures [13]. While the top-down approach focuses on breaking graphene precursor (graphite) into atomic layers from a stack (Fig. 1), bottomup approach implements carbon molecules as building blocks; typically these carbon molecules are obtained from alternative sources (Fig. 2). Even though the bottom-up approach is not suitable for the production of graphene sheets with large surface area, this approach offers the possibility of manufacturing graphene nano-ribbons and graphene dots (so-called nanoflakes) in large quantities [14].

Initially after the discovery of graphene, the most common method exercised was the micromechanical cleavage of graphite to synthesize high quality, defect-free graphene: this method is also called "scotch tape", drawing or peel off method [15]. Shortly after sometime, chemical vapour deposition (CVD) was used as a viable alternative [16, 17]. However, it was soon realised that these procedures offer a low production yield and particularly in the case of micromechanical cleavage they were time consuming that hindered effective and full-exploitation of these materials [18]. Even though researches have fine-tuned the technique to provide high quality crystallites up to $100 \mu \mathrm{m}$ in size, making it better separated (Fig. 1) in intercalated graphite compounds (socalled GIC) [1].

Another route alternative to the micromechanical cleavage is exfoliation of graphite or its derivatives, mainly graphite oxide (GO), which makes high production yield achievable, making the process cost-effective and scalable [19]. Exfoliation procedures have been scaled up and now companies are able to sell graphene in large quantities [20]. These are examples of the top-down 
approaches of graphene synthesis, where precursors of graphene are stripped down to few layers of graphite by chemical, mechanical or thermal treatments.

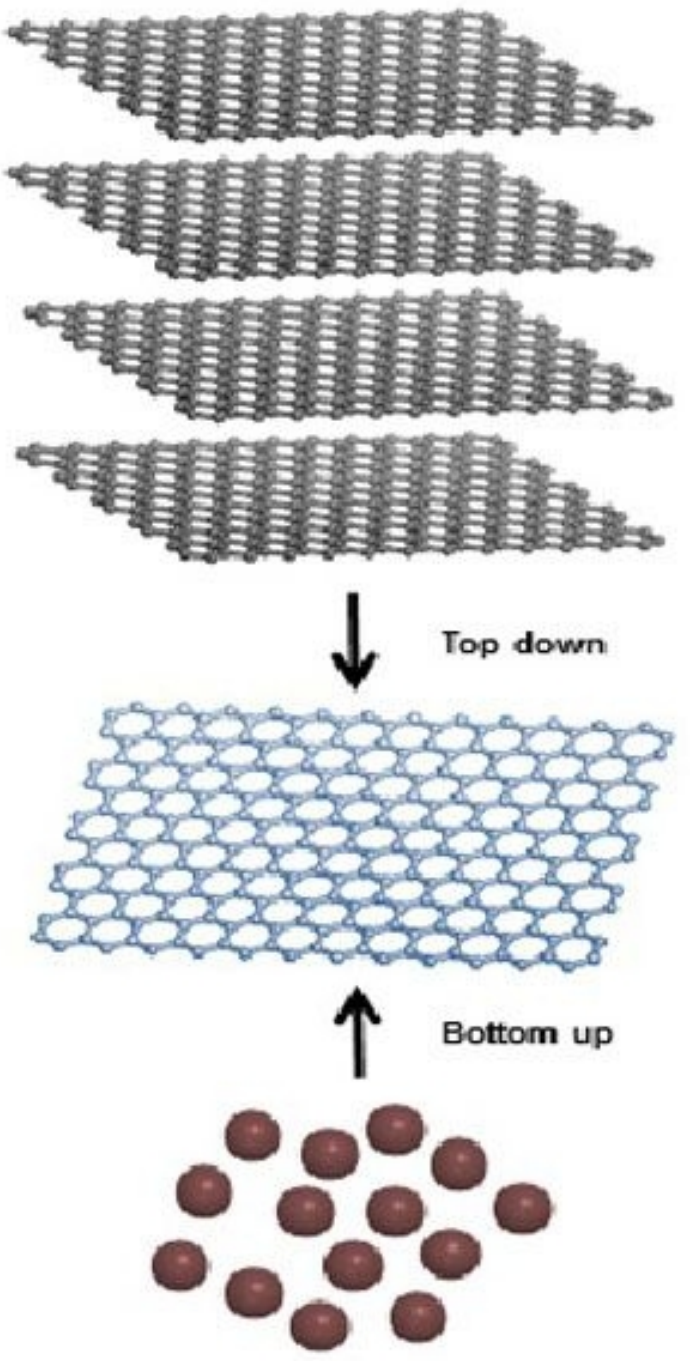

Fig. 2. Schematic showing bottom-up and top-down graphene synthesis approaches.

\subsection{Top-down approach}

\subsubsection{Mechanical exfoliation}

Mechanical or micromechanical exfoliation method has been a turning point in the history of graphene. It is still the key synthesis technique for obtaining high quality graphene for research purposes since it delivers high quality graphene films sized from 5 to $10 \mu \mathrm{m}$ as it has been witnessed by TEM and AFM analysis (Fig. 1). However, the uneven thickness of films obtained by this method and high production cost due to low yield render this method unusable for mass production. These methods are a prime example of the top-down approach, where a graphene precursor (graphite, GO, etc.) is taken apart layer by layer, forming graphene sheets. The group from Manchester University including the two Nobel Prize winners, Geim and Novoselov, obtained graphene by micro-mechanical alleviation of graphite. They used 'the scotch tape' or peel off method to repeatedly split graphite crystals into increasingly thinner pieces. The tape containing optically transparent flakes was then dissolved in acetone; a few steps later, the flakes containing both multi-layer and monolayer graphene were sedimented on a silicon wafer to be studied under a microscope. This technique was later modified by researchers to avoid the stage, where graphene floated in a liquid. Such developments eventually led to graphene flakes with sizes larger than $1 \mathrm{~mm}$ which were visible to the naked eye [21]. Ability to isolate graphene caused the current research boom. Prior to it, free-standing atomic planes were thought not to exist [15]. It was widely believed by scientists that such structures would be unstable, if scaled down to nanometers and were expected to scroll and buckle [22, 23]. Some scientists also believe that the intrinsic microscopic roughening on the scale of $1 \mathrm{~nm}$ might play a role in the stability of 2D crystals [24].

Novoselov et al. [26] applied similar experimental approaches to other materials obtaining free-standing atomic planes of boron nitride, mica, dichalcogenides and complex oxides, but none of them have managed to achieve as much attention so far. Jayasena et al. [27] reported another approach towards mechanical exfoliation which used a diamond wedge to literally scrape off graphene from a graphite source (highly ordered pyrolitic graphite, so-called HOPG), aided by ultrasonic oscillations. This, in principle, shares the same ideology as the approach observed by Geim et al., where layers of graphene are mechanically removed from the carbon source. However, due to the ability to control frequency of oscillations 


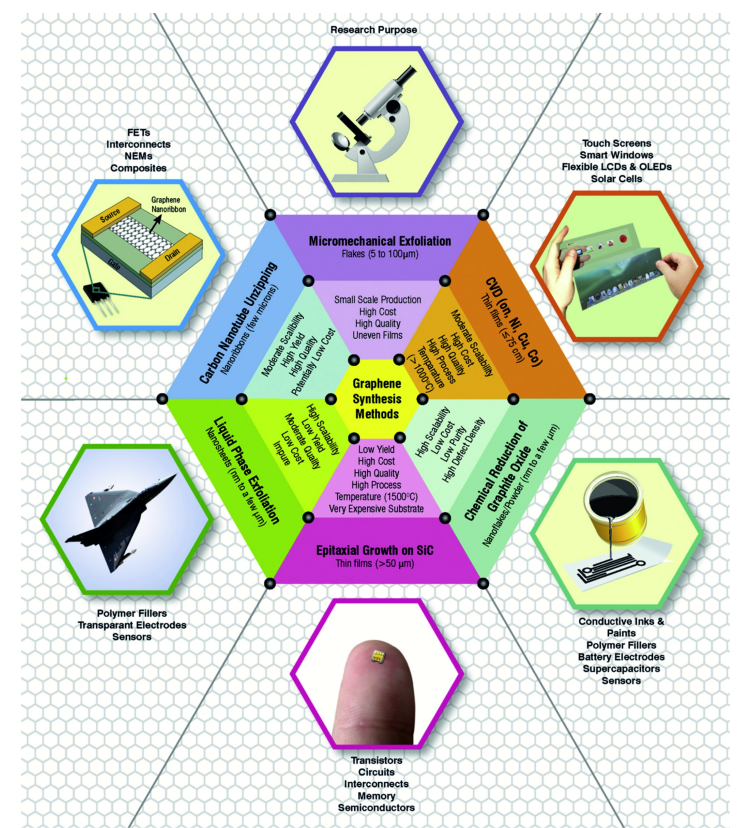

Fig. 3. Schematic of some popular graphene synthesis techniques along with their respective features, and their potential applications [25]. (re-printed with permission).

and contact pressure, this allows more control resulting in consistent properties of thin layer graphene. As highlighted by Jayasena et al., this approach requires better understanding of the relation between the edge formation and ultrasonic vibrations to be able to explain nanohorns-like structure formation around the edges. It should also be interesting to study localized temperature increase caused by friction. This method has the potential to provide high quality graphene for experimental purposes, but does not seem suitable for being scaled up. Keith et al. have recently reported graphene synthesis by shear exfoliation in stabilizing liquids (N-methyl-2-pyrrolidone, sodium cholate, etc.) in which graphite is immersed and mixed at local shear rates higher than $10^{4} \mathrm{~s}^{-1}$. This approach attempts to replace the usage of sonication as means to separate layers, resulting in a less energy consuming and scalable approach. It also allows production of defect-free and un-oxidized graphene. As noted by Keith et al., shearing rate must be higher than $\sim 10^{4} \mathrm{~s}^{-1}$, which is achievable in a simple kitchen blender, too. Graphene obtained by this method, in terms of properties, is identical to what is achievable by sonication of graphite in solvents and surfactants. Because of the simplicity of this method, it seems viable for industrial scale manufacturing of graphene. Interestingly, this method can also be applied to exfoliate other layered materials, like boron nitride and molybdinum disulfide [28]. An additional step to help improve the quality of graphene obtained by GO, which, as a precursor, can be obtained by many methods, has been proposed by Zhang et al. who has chosen the method reported by McAllister et al. [29] and treated it under a hot press at $1500{ }^{\circ} \mathrm{C}$ for $5 \mathrm{~min}$, under uniaxial pressures of $40 \mathrm{MPa}$. GO sheets start to get reduced to graphene sheets between $800{ }^{\circ} \mathrm{C}$ to $1500{ }^{\circ} \mathrm{C}$. This method can help obtain gram-scale highly crystalline graphene. High temperature and pressure treatment of GO sheets offer a step further from thermal treatment of GO to reduce them into few layer graphene sheets. The addition of high pressure into the system further improves the proportion of GO sheets being reduced to graphene sheets. This technique also stands as a viable contender for being scaled up [30].

\subsubsection{Graphite intercalation}

Graphite can also be reduced to graphene via intercalation. Different chemical species can be inserted between graphite interlayer space to produce graphite intercalation compounds (GIC). Due to the presence of these intercalants between graphite layers, the distance between them increases. This also changes the properties of graphene because the increased interlayer distance affects electronic coupling between graphene layers. Different intercalants could lead to GIC having different set of properties usable for applications focusing on electrical, thermal and magnetic performance [31]. Lee et al. [32] have studied the effects of intercalation on capacitance in an electrode and have reported improved electro-conductivity of $\mathrm{MnO}_{2}$ due to the presence of GIC.

\subsubsection{Nanotube slicing}

Carbon nanotubes have also been used as a precursor for graphene. Graphene nanoribbons which are a few microns in size can be obtained by this 
approach [25]. Such methods offer high yield and good quality graphene. Theoretically, carbon nanotubes could be cut open to form 2D graphene sheets [33]. Several methods have been developed, like plasma etching of nanotubes partly embedded in polymer film [34] or placing carbon nanotubes in a solution containing potassium permanganate and sulfuric acid [35]. High quality graphene can be obtained from nanotube slicing. Chen et al. [36] developed a nanotube slicing technique which is suitable for industrial use. Carbon nanotubes were abraded between ground-glass surface under a constant load of $0.06 \mathrm{~N} / \mathrm{cm}^{2}$ causing friction to slice them into graphene sheets. Graphene obtained by this approach can be used in applications, such as FET, interconnects, NEM and composites [25]. However, given the novelty of the precursor (CNT), this method might not be financially viable for commercial production.

\subsubsection{Pyrolysis method}

Pyrolysis offers ease of process and can be scaled up for production. However, the yield is low and there are impurities in the final product. In this method, a 1:1 molar ratio of sodium $(2 \mathrm{~g})$ and ethanol $(5 \mathrm{~mL})$ are heated in a sealed reactor vessel at $220{ }^{\circ} \mathrm{C}$ for 72 hours to obtain the graphene precursor: solid solvothermal product. It is then rapidly pyrolysed and the remaining product washed with deionized water $(100 \mathrm{~mL})$. The suspended solid is then vacuum filtered and dried in a vacuum oven at $100{ }^{\circ} \mathrm{C}$ for 24 hours. This solvothermal reaction method can deliver graphene yield of approx. $0.1 \mathrm{~g}$ per $1 \mathrm{~mL}$ of ethanol-typically yielding $0.5 \mathrm{~g}$ per reaction [37].

Alternative methods: Pyrolysis has also been used to obtain carbon forms bearing high energy density for super capacitor application; interestingly these were derived from biomass and their properties bear resemblance with graphene [38].

\subsubsection{Reduction of graphite oxide (GO)}

Graphene nanoflakes/powder, from a $\mathrm{nm}$ to a few $\mu \mathrm{m}$ in size, is obtainable by chemical reduction of graphite oxide (GO). Graphene obtained by this method is suitable for use in conductive inks and paints, polymer fillers, battery electrodes, supercapacitors, sensors, etc. Reduction of GO has generally been preferred for graphene reduction because of the lower degree of exfoliation achieved by graphite and expandable graphite [39-41]. GO can be exfoliated by sonication in water but the resulting material is electrically insulating, so it should be reduced to restore its conductivity. Reduction can be performed by chemical or thermal treatment [18]. Rapid heating has also been reported as a possible solution resulting in high yields of dispersed carbon powder with a few percent of carbon flakes [42]. Also, by dispersing oxidized and chemically processed graphite in water, and using paper-making techniques, the monolayer flakes in a form of a single sheet bonded very powerfully can be obtained. These sheets, called graphene oxide paper, have a measured tensile modulus of $32 \mathrm{GPa}$ [43]. Graphene based membranes are impermeable to all gases and liquids except water, which evaporates through the membrane, like it did not exist [44]. GO reduction under ambient atmosphere with the aid of $\mathrm{HCl}$ has also been reported [45]. Gurunathan et al. have developed a novel method of GO reduction with the help of biomass obtained from lysogeny broth bacteria. In their reported method, bacteria grown from lysogeny broth was incubated for 21 hours in an Erlenmeyer flask, at $120 \mathrm{rpm}$ and $37^{\circ} \mathrm{C}$. The resultant culture was centrifuged at $10000 \mathrm{rpm}$ to obtain the biomass used for rGO synthesis. $200 \mathrm{mg}$ of Bacillus marisflavi biomass was added to the $\mathrm{GO}$ dispersion $(0.5 \mathrm{mg} / \mathrm{mL})$ and stirred for $72 \mathrm{~h}$ at $37{ }^{\circ} \mathrm{C}$, followed by centrifugation to remove excess bacteria [46]. While chemical methods make it possible to synthesize graphene at lower temperatures and cost, graphene obtained by such methods contains high density of surface defects. Graphene obtained from this approach is also of a low purity.

\subsubsection{Electrochemical exfoliation}

Parvez et al. reported an interesting method of exfoliating graphene from graphite using an electrochemical method. They used $0.1 \mathrm{M}$ $\mathrm{H}_{2} \mathrm{SO}_{4}$ solution as electrolyte, while graphite flakes as anode and platinum wires as cathode. A positive charge of $+10 \mathrm{~V}$ was used to the system and graphite flakes began to dissolve in the 
solution. After 2 minutes, the voltage bias was removed and the exfoliated graphitic material was collected by vacuum filtration. The sample was then washed with water repeatedly to ensure removal of residual acidic content. The obtained powder was dispersed in DMF, resulting in EG sheets [47]. Lu et al. have proposed a one-pot method to synthesize different nanocarbon forms, including graphene, by exfoliation of graphite in ionic liquids. They experimented with incorporation of 1-butyl-3-methylimidazolium tetraflouroborate $[\mathrm{BMIm}]\left[\mathrm{BF}_{4}\right]$ in water used as an electrolyte for the electrochemical exfoliation of graphite. Static potentials of 1.5 to $15 \mathrm{~V}$ were applied. The exfoliated particles were washed with water and ethanol to achieve a neutral $\mathrm{pH}$ [48].

\subsubsection{Sonication}

High quality, un-oxidized graphite and graphene flakes can be obtained by sonication [49]. This method is a prime example of the top-down approach towards graphene synthesis since, in this method, ultrasonic energy is used to separate graphene layers stacked together in a precursor. But this method requires a large amount of energy since sonication is the only energy source and it can be a concern, while scaling up this method [27]. Also, removal of impurities is a problem that occurs in this process. Graphene obtained by this technique can be used in the field of polymer fillers, transparent electrodes and sensors [25]. Graphene can be dispersed in certain solvents, like $\mathrm{N}$-methyl-pyrrolidone, at concentrations of up to $0.01 \mathrm{mg} / \mathrm{mL}$. Such solutions can be used in a variety of processes to incorporate graphene in polymers and composites, such as spray coating, vacuum filtration, solvent casting or drop casting [49]. While sonication presents an easy approach towards making graphene, it poses challenges for industrialization in terms of power consumption. Solvent aided sonication is a simple modification of sonication method and an obvious step ahead. Graphene is obtained from graphite through sonication with the help of a solvent (NMP, TEA, etc.), where graphene can be separated from graphite with the help of centrifugation [49]. NMP or other ionic liquid can be used in such process [50, 51]. A singlecycle yield of up to $5 \%$ is obtainable from this process and about $70 \%$ of the graphene obtained by this process is $1 \mathrm{~nm}$ thick having electrical conductivity of up to $5000 \mathrm{~S} \cdot \mathrm{m}^{-1}$ [52]. When using the solvent aided systems, graphene tends to re-stack together after sonication because of van der Waals forces. To avoid such problems, surfactants or dispersing agents can be added in the solution before sonication, so the graphene sheets would not re-stack. This method can be used to obtain graphene sheets from graphite without chemical modification. It also offers graphene yields as high as $5.33 \mathrm{mg} / \mathrm{mL}$. Because of the use of an ionic liquid (1-hexyl-3-methyl-imidazolium hexafluorophosphate or $\mathrm{HMIH}$ ), this process is stable and green [51]. Sodium dodecylbenzene sulfonate (SDBS) also tends to stabilize graphene layers and keeps them from aggregation [53]. Liu et al. have reported a method using GO suspension (via sonication) to obtain 3D hydrogel as a support for microbe immobilization and BOD biosensor. Neutral Red (NR) solution was added into $20 \mathrm{~mL}$ of $2 \mathrm{mg} / \mathrm{mL}$ GO suspension, which was sonicated further for $10 \mathrm{~min}$ and then the solution was kept at $180{ }^{\circ} \mathrm{C}$ for $5 \mathrm{~h}$ in a teflon-lined autoclave, followed by temperature reduction to room temperature. The obtained hydrogel was washed thoroughly with deionized water before characterization [54].

$\pi-\pi$ interaction has a great potential to be used as an assistance in this regard. Aromatic ring containing organic molecules could be used to prevent graphene from restacking [55-59]. It is also possible to use the high energy interface between two immiscible liquids to hold the graphene sheets after sonication, thus, prevent them from re-stacking. Heptane and water are a viable couple to be used as immiscible liquids. Graphene sheets obtained in this process are up to $95 \%$ transparent and conductive. This process allows obtaining macroscopic graphene coatings on glass surfaces with the help of chlorosilanes, containing no more than 3 layered graphene stacks [60]. This method has a great potential for use in optoelectronics and solar panels. GO can also be reduced to graphene sheets via sonochemical treatment. The $\mathrm{pH}$ of GO solution is 
modified to be 10 by adding $\mathrm{NaOH}$ and a small amount of hydrazine. The solution should then be subjected to sonication for $2 \mathrm{~h}$. Because the temperature is unregulated in this method, the solution might reach temperatures of up to $65^{\circ} \mathrm{C}$ by the end of sonication [12, 61-64]. Hydrazine reacting with $\mathrm{GO}$ in the presence of ultrasonic irradiation causes acoustic cavitation resulting in more reactive sites for epoxide and hydroxyl removal. Also, the temperature induced by the irradiation itself causes reduction of GO into graphene since GO is not thermally stable above $200{ }^{\circ} \mathrm{C}$ [12].

\subsubsection{Ball milling}

In principle, ball milling is a sub-category of mechanical exfoliation but many variations of this method have been developed, so this method deserves to be discussed separately. Ball milling has been in use for mixing and particle size reduction for a long time. Scientists have used this particle size reduction approach to exfoliate graphene sheets from graphite, sometimes using magnetic assistance [65] and sometimes using chemical [66, 67] or solvent assistance [68]. Magnetic assistance provides filtration of metallic particles present in the precursors, while chemical assistance prevents the sheets from re-stacking using chemical interactions, such as $\pi-\pi$ [66]. Such techniques provide FLGs ( $\leqslant 5$ layers) and offer high production yield. Compared to graphite, a larger surface area of graphene $\left(153.9 \mathrm{~m}^{2} / \mathrm{g}\right)$ can be obtained using this process [69] and impressively low $\mathrm{I}_{\mathrm{D}} / \mathrm{I}_{\mathrm{G}}$ ratio of 0.6 can be obtained [66].

\subsubsection{Radiation based methods}

Radiation based methods offer graphene production in a relatively short period of time. High quality graphene is obtainable by this method but the yield is low. Also, we believe radiation concerns will not allow such methods to be used industrially regardless of the fact that this process is financially not viable for industrialization. GO can also be reduced to graphene sheets using electron beam irradiation. According to the reported method, an aqueous dispersion of GO and isopropyl alcohol was put in a sealed plastic bag and irradiated for $10 \mathrm{~min}$ under $2 \mathrm{MeV} / 10 \mathrm{~mA}$ conditions (140 kGy doses). The reduced GO was then separated by centrifugation, washed with alcohol a couple of times and dried at $60{ }^{\circ} \mathrm{C}$ under vacuum [70]. Laser scribing is a process similar in mechanism to pyrolysis; however, the energy source used here is a laser. GO is coated on a DVD which is then treated inside a LightScribe DVD drive to directly reduce GO films to graphene [71]. This technology has the potential to lead us to self-powering smart garments, outfitted with piezoelectric patches to harvest energy from body movements. Power source to camouflage uniforms is one possible application [72]. Other methods have been reported with the same phenomenon but with different light sources, such as UV or xenon flash [73, 74], or different kinds of lasers, such as femtosecond laser, pulsed laser, etc. [75-79]. In addition, Zhou et al. have reported a method to selectively reduce GO by laser writing [80].

\subsection{Bottom-up approach}

\subsubsection{Growth from metal-carbon melts}

In this simple method, a carbon source (e.g. graphite powder) is placed in contact with a transition metal and heated at high temperatures, enough to melt the metal. Once carbon starts to dissolve in the molten metal, temperature can be lowered to decrease solubility of carbon resulting in excessive carbon precipitation. The precipitate can be skimmed off to give different forms of carbons, e.g. thick graphite, few layers of graphene (FLG) and single layers of graphene (SLG). Nickel seems most suited for this process since it is not Raman active [81]. Ruthenium and iridium are known to be usable too but the quality of graphene is not as good as what is achievable from nickel and copper $[82,83]$. There have been several different variations of this technique reported [81, 84]. However, the most appreciated method seems to be graphene growth on nickel films using CVD. Nickel film is heated up to 900 to $1000{ }^{\circ} \mathrm{C}$ under inert conditions (Ar gas used). Methane gas is introduced into the system along with Ar. This enables the carbon from the methane gas to be absorbed on the nickel film. Methane supply is later discontinued and the 
system is allowed to cool down (under Ar flow). This enables the carbon to diffuse out of nickel surface and form graphene sheets [85-88]. Similar to nickel based method, this method uses $\mathrm{Cu}$ substrate and methane gas depositing carbon on the surface, under high temperature and low pressure. However, atmospheric pressure leads to multilayer graphene on both copper and nickel [89]. It is possible to grow large films of graphene using this method [87, 90, 91]. In contrast to methane gas, ethane and propane tend to produce bilayer coatings; this could be due to their molecular structure containing a higher concentration of carbon [92].

\subsubsection{Epitaxial growth on silicon carbide (SiC)}

Graphene can be epitaxially grown on $\mathrm{SiC}$ substrate ideal to be used in transistors and circuits because of the thin graphene films obtainable by this method $(>50 \mu \mathrm{m})$. In this method, graphene is obtained by heat treatment of silicon carbide ( $\mathrm{SiC})$ at $1100{ }^{\circ} \mathrm{C}$ [93]. This method leads to obtaining epitaxial graphene, while the size of graphene flakes remains dependent upon the size of SiC wafers. The surface of SiC influences the thickness, mobility and carrier density of graphene obtained in this system as observed in research [94]. Graphene obtained from this procedure tends to have weak anti-localization, unlike exfoliated graphene [95]. On the other hand, similar to graphene obtained by drawing or peel off method, SiC-epitaxial graphene displays extremely large, temperature independent mobility but not as high as exfoliated graphene [96]. Even without transfer, graphene obtained through this method displays massless Dirac fermions [85, 96101]. In multi-layered epitaxial graphene, weak van der Waals forces responsible for multilayer cohesion do not necessarily impact electronic properties of individual sheets within a stack. This effect is related to the symmetry of interlayer interactions [102]. This phenomenon does not occur in other cases and electronic properties are affected, e.g. in bulk graphite. A 2-inch $\mathrm{SiC}$ wafer can offer cut off frequencies of $100 \mathrm{GHz}$ [103]. This technique offers high quality graphene but at high production cost because $\mathrm{SiC}$ substrate is expensive and the low yield is obtained. So this method is not suitable for industrial manufacturing.

\subsubsection{Dry ice method}

According to dry ice method, graphene can be obtained by burning $3 \mathrm{~g}$ of $\mathrm{Mg}$ ribbon inside a dry ice bowl, covered up by another dry ice $\left(\right.$ solid $\left.\mathrm{CO}_{2}\right)$ slab. $\mathrm{Mg}$ needs to be completely burnt in $\mathrm{CO}_{2}$ and the residue is stirred overnight in $100 \mathrm{~mL}$ of $1 \mathrm{M}$ $\mathrm{HCl}$. Both $\mathrm{Mg}$ and $\mathrm{MgO}$ are soluble in water. So, the mixture is filtered and the residue is washed with deionized water till $\mathrm{pH}$ turns neutral. To remove water content, the residue needs to be dried overnight under vacuum at $100{ }^{\circ} \mathrm{C}$ resulting in a graphene yield of $680 \mathrm{mg}(92 \%)$ [104].

\subsubsection{Deposition}

There have been a lot of methods reported for deposition-based graphene synthesis, primarily because of its practicality and ease of scalability [47, 105-110]. Chemical Vapor Deposition (CVD) techniques lead to thin graphene films $(\leqslant 75 \mathrm{~cm})$ [25]. Because of the large size of these films, they are suitable for use in applications, such as touch screens, smart windows, solar cells, flexible LCD and OLED. CVD methods enable growth of good quality, large area graphene films [17, 85, 90]. Most of the CVD techniques are scalable but are financially not viable. Spin coating enables nanotube-reinforced graphene to be made. Dodecyl-functionalized single wall carbon nanotubes (DF-SWCNT) are used in this process via spin coating and annealing, providing stronger, flexible and more conductive graphene. HIP co-produced SWCNT were used in the reported method [111]. DF-SWCNT and trichloromethane solutions were made as reported by Liang et al. [112]. Solution containing DFSWCNT and trichloromethane was dispersed on a pre-treated $\mathrm{Cu}$ foil using spin-coating which was then annealed for $15 \mathrm{~min}$ at $1080{ }^{\circ} \mathrm{C}$ under $\mathrm{H}_{2}$ and Ar flow, while keeping the chamber pressure constant at 933.25 $\mathrm{Pa}$. The samples were reported to be removed and allowed to cool down to room temperature afterwards [113]. Supersonic spray offers a simple and inexpensive option to deposit graphene films on to a variety of substrates, in contrast 
to CVD. This method does not actually synthesize graphene but improves it. With this treatment, graphene with little or no defects can be obtained, having $\mathrm{I}_{\mathrm{D}} / \mathrm{I}_{\mathrm{G}}$ ratios as low as 0.22 [114]. This system uses kinetic spray deposition that benefits from the supersonic acceleration of droplets through a converging and diverging de Laval nozzle [47]. R-Go gets atomized upon exposure to high gas (air) stream [114]. In dip coating, GO is deposited on a substrate (typically, silica or quartz) and then reduced into graphene via thermal treatment under continuous $\mathrm{Ar}$ or $\mathrm{H}_{2}$ flow. This method allows better control of film thickness [115]. This method can also be beneficial for producing solar panels and other optoelectronic applications. It offers excellent conductivity and optical transparency in visible and near-infrared regions, chemical and thermal stability along with the benefit of transferability between substrates. Vacuum filtration can lead to graphene films by filtering GO suspension through a cellulose ester membrane with an average pore size of $25 \mathrm{~nm}$. As the solvent drains out, the GO forms a layer on the cellulose substrate. Since GO tends to block pores, it does not allow further passage of the solvent once a pore is filled, what leads the remaining GO suspension to other (vacant) pores, making this a self-regulating system. The thickness of these layers can be controlled by the amount of solution or its concentration. Once filtration is completed, the substrate (cellulose) layer can be dissolved by placing the film sandwich sideways in acetone (or other suitable solvents) because the GO film obtained by this process can be used in lithographic processes. Once an independent GO film is obtained, it requires to be reduced to graphene films [106]. Several methods have been reported to perform this operation, including the method in which ordered nano and macroscale FLG is exfoliated from a pre-patterned and pristine HOPG surface and printed on a semiconductor substrate by an electrostatic force, reported by Liang et al. [112]. However, a combination of hydrazine vapor exposure and low-temperature annealing treatment renders efficient results [117, 118]. Li et al. have reported a method to synthesize multilayer films based on graphene and manganese dioxide $\left(\mathrm{MnO}_{2}\right)$ sheets using electrostatic layer-by-layer deposition. Successful transfer and creation of multilayer films were obtained by dipping indium tin oxide (ITO) coated glass slides in poly(diallyldimethylammonium) (PDDA) aqueous solution ( 1 wt.\%, $\mathrm{pH}=9.37$ ), to have the substrate positively charged. The sheets were kept in the solution for $15 \mathrm{~min}$. To remove the excess solution, the sheets were rinsed with ultrapure water and dried under continuous nitrogen flow. The obtained substrates, coated and positively charged, were then immersed in a poly(sodium 4styrenesulfonate) modified graphene sheets (PSSGS) solution $\left(1 \mathrm{mg} \cdot \mathrm{mL}^{-1}, \mathrm{pH}=9.6\right)$ for $20 \mathrm{~min}$, followed by washing and drying. This created a uniform layer of PSS-GS on the substrate. In order to obtain multilayers, the PSS-GS coated substrate was positively charged again in PDDA aqueous solution, creating another layer of PDDA just as the initial layer. To create a $\mathrm{MnO}_{2}$ layer, the substrates were dipped in a colloidal suspension of $\mathrm{MnO}_{2}$ nanosheets $\left(0.2 \mathrm{~g} \cdot \mathrm{L}^{-1}, \mathrm{pH}=9.15\right)$ followed by washing and drying. The final multilayer sheets of ITO/ $\left(\mathrm{PDDA} / \mathrm{PSS}-\mathrm{GS} / \mathrm{PDDA} / \mathrm{MnO}_{2}\right)_{\mathrm{n}}$ is suitable for use in supercapacitors [119].

Table 1. Comparison of intensity ratios ( $D$ and $G$ bands) between graphene obtained by different techniques.

\begin{tabular}{ccc}
\hline Method & $\mathrm{I}_{\mathrm{D}} / \mathrm{I}_{\mathrm{G}}$ & Reference \\
\hline \hline Wedged based exfoliation & $>0.6$ & {$[27]$} \\
Shearing & 0.18 & {$[28]$} \\
Hot Press Method & $>1.2$ & {$[30]$} \\
Nanotube Slicing & 0.704 & {$[36]$} \\
Nickel Based & 0.24 & {$[84]$} \\
Dry Ice Method & 0.84 & {$[104]$} \\
Electrochemical Exfoliation & 0.4 & {$[47]$} \\
Solvent aided Sonication & 0.85 & {$[51]$} \\
Rebar Graphene & 0.1 & {$[113]$} \\
Supersonic Spray & 0.22 & {$[114]$} \\
Dip Coating & 0.81 & {$[115]$} \\
Vacuum Filtration & 0.85 & {$[117]$} \\
Electron Beam Irradiation & 1.51 & {$[70]$} \\
\hline
\end{tabular}

Table 1 shows a comparison of D and $\mathrm{G}$ band intensity ratios. The intensity ratio or $\mathrm{I}_{\mathrm{D}} / \mathrm{I}_{\mathrm{G}}$ is a measure of disorder degree and average size of the 
sp2 domains [70]. It can also be used to calculate the crystallite size of graphene (or graphite) samples using Tuinstra-Koenig relation [120]. Lower degree of structural defects in graphene leads to smaller $\mathrm{I}_{\mathrm{D}} / \mathrm{I}_{\mathrm{G}}$ numbers.

\section{Conclusions}

Graphene has gained an immense amount of fame and scientific attention from the time of its inception. This is primarily because of its extraordinary properties and the potential it carries for high performance composites. Up-scaling graphene production is a challenge no less than synthesis of high quality graphene itself since many methods are not suitable for scaling up. Scientists have chosen different approaches to synthesize graphene using top-down and bottom-up approaches. Bottomup approaches, like CVD, provide excellent control of thickness and quality, while being unsuitable for industrialization. However, it can be used for producing graphene for high-end applications, such as touch screen LCD, OLED, solar panels, etc. In contrast to bottom-up approach, top-down approach comprises of breaking down graphene precursors, such as graphite, GO, etc. into few layer graphene. Many different techniques have surfaced so far to achieve this goal. Methods, such as shearing, provide easy and scalable approach. Graphene is being synthesized on an industrial scale [121], however, currently available methods rely on batch production and offer problems associated with it, for example difficulty in controlling consistency of properties which is vital for industrial scale production. We believe graphene might not be a 'one size fits all' case, where one particular kind of graphene would be suitable for all applications which is one of the reasons why graphene synthesized by different methods is suitable for use in different applications. Since new methods to synthesize graphene keep getting more and more interesting, we hope to see a solution that provides not just a fair balance between scalability and quality, but the best of both. The aim of this article is to provide an insight into graphene synthesis technology by providing an introduction and comparative study.

\section{References}

[1] Geim A.K., Novoselov K.S., Nat Mater, 6 (2007), 183.

[2] Allen M.J., Tung V.C., Kaner R.B., Chem. Rev., 110 (2009), 132.

[3] ENoki T., Suzuki M., ENdo M., Graphite Intercalation Compounds and Applications, Oxford University Press, New York, 2003.

[4] Delhaes P., Graphite and precursors, CRC Press, Amsterdam, 2001.

[5] Boehm H.P., Setton R., Stumpr E., Pure. Appl. Chem., 66 (1994), 1893.

[6] Castillo-Martinez E., Carretero-Gonzalez J., Sovich J., Lima M.D., J. Mater. Chem. A, 2 (2014), 221.

[7] Pauling L., The nature of the chemical bond and the structure of molecules and crystals: an introduction to modern structural chemistry, Cornell University Press, Ithaca (NY), 1960.

[8] Geim A.K., Science, 324 (2009), 1530.

[9] Kotov N.A., Nature, 442 (2006), 254.

[10] Rao C., Biswas K., Subrahmanyam K., GovinDARAJ A., J. Mater. Chem., 19 (2009), 2457.

[11] Soldano C., Mahmood A., Dujardin E., Carbon, 48 (2010), 2127.

[12] Krishnamoorthy K., Kim G.-S., Kim S.J., Ultrason. Sonochem., 20 (2013), 644.

[13] Edwards R.S., Coleman K.S., Nanoscale, 5 (2013) 38.

[14] Warner J.H., SChäFFEl F., BAChMatiuK A., RÜMMELI M.H., Graphene: Fundamentals and emergent applications, Elsevier, Oxford, 2012.

[15] Novoselov K.S., Geim A.K., Morozov S., JiAng D., Zhang Y., Dubonos S., Science, 306 (2004), 666.

[16] Dato A., Radmilovic V., Lee Z., Phillips J., FRENKLACH M., Nano Lett., 8 (2008), 2012.

[17] Reina A., Jia X., Ho J., Nezich D., Son H., BulOVIC V., Nano Lett., 9 (2008), 30.

[18] Verdejo R., Bernal M.M., Romasanta L.J., LoPEZ-MANCHADO M.A., J. Mater. Chem., 21 (2011), 3301.

[19] PARK S., RuofF R.S., Nat. Nanotechnol., 4 (2009), 217.

[20] SEgAL M., Nat. Nanotechnol., 4 (2009), 612.

[21] Geim A.K., Macdonald A.H., Phys. Today, 60 (8) (2007), 35.

[22] Shenderova O., Zhirnov V., Brenner D., Crit. Rev. Solid State, 27 (2002), 227.

[23] Sakamoto J., Heijst van J., Lukin O., SCHLÜTER A.D., Angew. Chem. Int. Edit., 48 (2009), 1030.

[24] MeYer J.C., GeIM A.K., Nature, 446 (2007), 60.

[25] Mittal G., Dhand V., Rhee K.Y., Park S.-J., LEE W.R., J. Ind. Eng. Chem., 21 (2015), 11.

[26] Novoselov K., Jiang D., Schedin F., Booth T., Khotkevich V., Morozov S., P. Natl. Acad. Sci. USA, 102 (2005), 10451. 
[27] Jayasena B., Subbiah S., Nanoscale Res Lett., 6 (2011), 95.

[28] Paton K.R., Varrla E., Backes C., Smith R.J., KHAN U., Nat. Mater., 13 (2014), 624.

[29] McAllister M.J., Li J.-L., Adamson D.H., Schniepp H.C., ABdala A.A., LiU J., Chem. Mater., 19 (2007), 4396.

[30] Zhang Y., Li D., TAN X., Zhang B., RuAn X., LiU H., Carbon, 54 (2013), 143.

[31] Zhan D., Sun L., Ni Z.H., LiU L., FAn X.F., WANG Y., Adv. Funct. Mater., 20 (2010), 3504.

[32] LeE H., KAng J., Cho M.S., Choi J.-B., LeE Y., J. Mater. Chem., 21 (2011), 18215.

[33] Brumfiel G., Nature, 10 (2009), 1038.

[34] Jiao L., Zhang L., Wang X., Diankov G., Dai H., Nature, 458 (2009), 877.

[35] Kosynkin D.V., Higginbotham A.L., Sinitskit A., Lomeda J.R., Dimiev A., Price B.K., Nature, 458 (2009), 872.

[36] Chen J., Chen L., Zhang Z., Li J., Wang L., JIANG W., Carbon, 50 (2012), 1934.

[37] Choucair M., Thordarson P., Stride J.A., Nat. Nanotechnol., 4 (2008), 30.

[38] Biswal M., Banerjee A., Deo M., Ogale S., Energ. Environ Sci., 6 (2013), 1249.

[39] Chen G., Wu D., Weng W., Wu C., Carbon, 41 (2003), 619.

[40] Ramanathan T., Stankovich S., Dikin D., LiU H., Shen H., Nguyen S., J. Polym. Sci. Pol. Phys., 45 (2007), 2097.

[41] Dreyer D.R., Park S., Bielawski C.W., RuofF R.S., Chem. Soc. Rev., 39 (2010), 228.

[42] Eswaraiah V., Aravind S.S.J., Ramaprabhu S., J. Mater. Chem., 21 (2011), 6800.

[43] Dikin D.A., Stankovich S., Zimney E.J., Piner R.D., Nature, 448 (2007), 457.

[44] NAIR R., Wu H., JAYARAM P., Grigorieva I., GEIM A., Science, 335 (2012), 442.

[45] Shen B., Lu D.D., Zhai W.T., Zheng W.G., J. MATER. ChEM. C, 1 (2013), 50.

[46] Gurunathan S., Han J.W., Eppakayala V., Kim J.-H., Int. J. Nanomed., 8 (2013), 1015.

[47] Parvez K., Li R., Puniredd S.R., HernandeZ Y., Hinkel F., WANG S., ACS Nano, 7 (2013), 3598.

[48] LU J., YANG J.-X., WANG J., Lim A., WANG S., LOH K.P., ACS Nano, 3 (2009), 2367.

[49] Hernandez Y., Nicolosi V., Lotya M., Blighe F.M., Sun Z., DE S., Nat. Nanotechnol., 3 (2008), 563.

[50] Alzari V., Nuvoli D., Scognamillo S., PicCinini M., Gioffredi E., Malucelli G., J. Mater. Chem., 21 (2011), 8727.

[51] Nuvoli D., Valentini L., Alzari V., ScogNAMillo S., Bon S.B., PicCinini M., J. Mater. Chem., 21 (2011), 3428.
[52] Zhou M., Tian T., Li X.F., Sun X.D., Zhang J., CUI P., Int. J. Electrochem. Sc., 9 (2014), 810.

[53] Lotya M., Hernandez Y., King P.J., Smith R.J., Nicolosi V., Karlsson L.S., J. Am. Chem. Soc., 131 (2009), 3611.

[54] LiU L., Zhai J., Zhu C., GaO Y., Wang Y., HaN Y., Biosens. Bioelectron., 63 (2015), 483.

[55] Xu Y., Bai H., Lu G., Li C., Shi G., J. Am. Chem. Soc., 130 (2008), 5856.

[56] Hao R., Qian W., Zhang L., Hou Y., Chem. Commun., 48 (2008), 6576.

[57] Patil A.J., Vickery J.L., Scott T.B., ManN S., Adv. Mater, 21 (2009), 3159.

[58] Englert J.M., RÖhrl J., Schmidt C.D., GraupNer R., Hundhausen M., Hauke F., Adv. Mater., 21 (2009), 4265.

[59] Su Q., Pang S., Alijani V., Li C., Feng X., MÜLLEN K., Adv. Mater., 21 (2009), 3191.

[60] Woltornist S.J., OYer A.J., CARrillo J.-M.Y., Dobrynin A.V., ADAMSON D.H., ACS Nano, 7 (2013), 7062.

[61] Deng C., Hu H., Ge X., Han C., Zhao D., Shao G., Ultrasonics., 18 (2011), 932.

[62] Pinjari D.V., Pandit A.B., Ultrasonics., 18 (2011), 1118.

[63] SAFARIFARD V., MORSAli A., Ultrasonics., 19 (2012), 823.

[64] Ramadoss A., Kim S.J., J. Alloy. Compd., 544 (2012), 115.

[65] LEE J.K., LEE K., LEE K.I., GAP L.J., IL L.G., Ball-milled graphene nano-powder or ribbon purifying method, involves separating magnetic impurities during stirring suspension using magnet, where impurities are incorporated into graphene powder during ball-milling, Korea Institute of Science and Technology, Seoul, p. 7.

[66] Leon V., Quintana M., Herrero M.A., Fierro J.L.G., Hoz de la A., Prato M., Chem. Commun., 47 (2011), 10936.

[67] Lin T., TANG Y., WANG Y., Bi H., LiU Z., HUANG F., Energ. Environ Sci., 6 (2013), 1283.

[68] Borah M., Dahiya M., Sharma S., Mathur R.B., Dhakate S.R., Mater. Focus, 3 (2014), 300.

[69] Liu L., Xiong Z., Hu D., Wu G., Chen P., Chem. Commun., 49 (2013), 7890.

[70] Pan D., Wang S., Zhao B., Wu M., Zhang H., WANG Y., Chem. Mater., 21 (2009), 3136.

[71] El-Kady M.F., Strong V., Dubin S., Kaner R.B., Science, 335 (2012), 1326.

[72] Miller J.R., Science, 335 (2012), 1312.

[73] Cote L.J., Cruz-Silva R., Huang J., J. Am. Chem. Soc., 131 (2009), 11027.

[74] Gao E., Wang W., Shang M., Xu J., Phys. Chem. Chem. Phys., 13 (2011), 2887.

[75] Abdelsayed V., Moussa S., Hassan H.M., Aluri H.S., Collinson M.M., El-Shall M.S., J. Phys. Chem. Lett., 1 (2010), 2804. 
[76] Huang L., Liu Y., Ji L.-C., Xie Y.-Q., Wang T., SHI W.-Z., Carbon, 49 (2011), 2431.

[77] Chichiov B., Momma C., Nolte S., ALvensleben Von F., Tünnermann A., Appl. Phys. A, 63 (1996), 109.

[78] Sokolov D.A., Shepperd K.R., Orlando T.M., J. Phys. Chem. Lett., 1 (2010), 2633.

[79] Trusovas R., Ratautas K., Račiukaitis G., Barkauskas J., StankevičIEnĖ I., Niaura G., Carbon, 52 (2013), 574.

[80] Zhou Y., Bao Q., Varghese B., Tang L.A.L., TAN C.K., Sow C.H., Adv. Mater., 22 (2010), 67.

[81] Amini S., Garay J., LiU G., Balandin A.A., ABBASChian R., J. Appl. Phys., 108 (2010), 094321.

[82] Sutter P.W., Flege J.-I., Sutter E.A., Nat. Mater., 7 (2008), 406.

[83] Pletikosić i., Kralu M., Pervan P., Brako R., CorauX J., N'DiAYE A., Phys. Rev. Lett., 102 (2009), 056808.

[84] Weatherup R.S., Bayer B.C., Blume R., Ducati C., Baehtz C., Schlögl R., Nano Lett., 11 (2011), 4154.

[85] Kim K.S., ZhaO Y., Jang H., LeE S.Y., Kim J.M., KIM K.S., Nature, 457 (2009), 706.

[86] Zhang Y., Zhang L., Zhou C., Accounts Chem. Res., 46 (2013), 2329.

[87] Bae S., Kim H., LeE Y., Xu X., Park J.-S., ZhenG Y., Nat. Nanotechnol., 574 (2010), 574.

[88] Rafiee J., Mi X., Gullapalli H., Thomas A.V., YAVARI F., Shi Y., Nat. Mater., 11 (2012), 217.

[89] Lenski D.R., Fuhrer M.S., J. Appl. Phys., 110 (2011), 013720.

[90] Li X., Cai W., An J., Kim S., Nah J., Yang D., Science, 324 (2009), 1312.

[91] Levendorf M.P., Ruiz-Vargas C.S., Garg S., PARK J., Nano Lett., 9 (2009), 4479.

[92] Wassei J.K., Mecklenburg M., Torres J.A., Fowler J.D., Regan B., Kaner R.B., Small, 8 (2012), 1415.

[93] SutTer P., Nat. Mater. 8 (2009), 171.

[94] Ohta T., Bostwick A., McChesney J., Seyller T., Horn K., Rotenberg E., Phys. Rev. Lett., 98 (2007), 206802.

[95] Morozov S., Novoselov K., Katsnelson M., Schedin F., Ponomarenko L., Jiang D., Phys. Rev. Lett., 97 (2006), 016801.

[96] Jobst J., Waldmann D., Speck F., Hirner R., MAude D.K., SEYller T., http://arxiv.org/ abs/0908.1900, 2009.

[97] Shen T., Gu J., Xu M., Wu Y., Bolen M., CAPANO M., Appl. Phys. Lett., 95 (2009), 172105.

[98] Wu X., Hu Y., Ruan M., Madiomanana N.K., Hankinson J., Sprinkle M., Appl. Phys. Lett., 95 (2009), 223108.

[99] Alexander-Webber J., BAKer A., JAnssen T., TZalenchuK A., Lara-Avila S., KubatKin S., Phys. Rev. Lett., 111 (2013), 096601.
[100] Tzalenchuk A., Lara-Avila S., Kalaboukhov A., PaOlillo S., Syväjärvi M., Yakimova R., Nat. Nanotechnol., 5 (2010), 186.

[101] Lara-Avila S., Kalaboukhov A., Paolillo S., SYVÄJÄrVi M., YAKIMOVA R., FAL'Ko V., arXiv:09091193, 2009.

[102] Hass J., VARChon F., Millan-Otoya J.-E., Sprinkle M., Sharma N., Heer de W.A., Phys. Rev. Lett., 100 (2008), 125504.

[103] Lin Y.-M., Dimitrakopoulos C., Jenkins K.A., FARMER D.B., CHIU H.-Y., Grill A., Science, 327 (2010), 662.

[104] Chakrabarti A., Lu J., Skrabutenas J.C., Xu T., Xiao Z., Maguire J.A., J. Mater. Chem., 21 (2011), 9491.

[105] Blake P., BRimicombe P.D., NAIR R.R., Booth T.J., JiAng D., Schedin F., Nano Lett., 8 (2008), 1704.

[106] Eda G., Fanchini G., Chhowalla M., Nat. Nanotechnol., 3 (2008), 270.

[107] Li D., Müller M.B., Gilje S., KANer R.B., WALLACE G.G., Nat. Nanotechnol., 3 (2008), 101.

[108] Rahaman M., Ismail A.F., Mustafa A., Polym. Degrad. Stabil., 92 (2007), 1421.

[109] Ko Y.U., Cho S.-R., Choi K.S., PARK Y., KIM S.T., KIM N.H., J. Mater. Chem., 22 (2012), 3606.

[110] Yamaguchi H., Eda G., Mattevi C., Kim H., ChHowalla M., ACS Nano., 4 (2010), 524.

[111] Nikolaev P., Bronikowski M.J., Bradley R.K., Rohmund F., Colbert D.T., SMith K., Chem. Phys. Lett., 313 (1999), 91.

[112] Liang F., Sadana A.K., Peera A., ChattopadhyAY J., Gu Z., HaUge R.H., Nano Lett., 4 (2004), 1257.

[113] Yan Z., Peng Z., Casillas G., Lin J., Xiang C., ZHOU H., ACS Nano, 8 (2014), 5061.

[114] Irissou E., Legoux J.-G., Ryabinin A., Jodoin B., Moreau C., J. Therm. Spray Techn., 17 (2008), 495.

[115] Wang X., Zhi L., Müllen K., Nano Lett. 8 (2008), 323.

[116] Liang X., Chang A.S.P., Zhang Y., Harteneck B.D., Choo H., Olynick D.L., Cabrini S., Nano Lett., 9 (1) (2009), 467.

[117] Stankovich S., Dikin D.A., Piner R.D., KohlhaAs K.A., Kleinhammes A., Jia Y., Wu Y., NGUYen S.T., RuOfF R.S., Carbon, 45 (7) (2007), 1558.

[118] Watcharotone S., Dikin D.A., Stankovich S., Piner R., Jung I., Dommett G.H.B., EvmeNENKo G., WU S.-E., Chen S.-F., LIU CH.,-P., NGUEN S.T., RuOFF R.S., Nano Lett., 7 (7) (2007), 1888.

[119] Li Z., WAng J., LiU X., LiU S., OU J., YAnG S.,, J. Mater. Chem., 21 (2011), 3397.

[120] Gómez-Navarro C., Weitz R.T., Bittner A.M., Scolari M., Mews A., Burghard M., Kern N., Nano Lett., 7 (11) (2007), 3499. 
[121] SHEN H., China's Graphene industry set to skyrocket in 2014, http://investorintel.com/graphite-grapheneintel/chinas-graphene-industry-starts-take-2014/,

Received 2014-11-17

2014.

Accepted 2015-05-13 